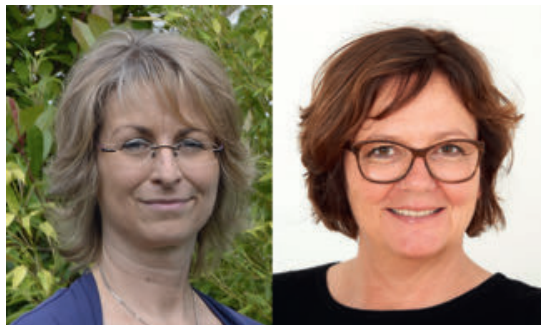

Auf Cordula Ahrendt folgt Heidi Bernard im Herausgeberteam. (Fotos: privat)

In eigener Sache

\section{Wechsel im Herausgeberteam}

Liebe Hebammen, liebe Leserinnen und Leser, beim letzten Herausgebertreffen hat sich Cordula Ahrendt aus dem Herausgeberteam verabschiedet, allerdings nicht ohne zu betonen, der Zeitschrift verbunden zu bleiben. Ein schwerer Abschied. Die Zeitschrift verliert eine erfahrene Herausgeberin, eine in berufs- und besonders ausbildungspolitischen Fragen sehr engagierte und kompetente Hebamme, eine tolle Kollegin. Aber niemals geht man so ganz. Cordula Ahrendt bleibt uns als Buch- und Zeitschriftenautorin erhalten und ist nun Mitglied des Fachbeirats der Zeitschrift. Wir, und da spreche ich sicher im Namen des gesamten Herausgeberund Redaktionsteams, danken Frau Ahrendt ganz herzlich für ihren jahrelangen Einsatz für DIE HEBAMME, die Hebammen und für den Verlag.

Gleichzeitig durften wir Heidi Bernard neu im Herausgeberteam willkommen heißen. Sie ist Hebamme, Lehrerin für Pflege und Gesundheit M.A. sowie Leiterin der Elternschule Neue Kölner in Köln. Sie bringt ab sofort ganz besonders die Perspektive selbstständiger Hebammen ein. Eine wunderbare Ergänzung und Verstärkung, über die wir uns sehr freuen. Wir wünschen Frau Bernard viel Freude bei ihrem neuen hochspannenden „Zusatz-Job“ als Herausgeberin.

Rosi Haarer-Becker

Projektleitung DIE HEBAMME und Programmbereichsleiterin Therapieberufe Thieme Gruppe
Neue Studienergebnisse

\section{Masernimpfung schützt auch vor anderen Infektionen}

Das Ende 2019 eingeführte Masernschutzgesetz sieht vor, dass alle Kinder ab dem vollendeten ersten Lebensjahr beim Eintritt in Kindergarten oder Schule die von der STIKO empfohlene Masernimpfung vorweisen müssen. Aktuell liefern zwei amerikanische Studien neue Argumente: Schon lange beobachten Mediziner, dass Kinder nach einer Masernerkrankung anfälliger für Infektionen sind. Epidemiologische Studien fanden unerklärbar erhöhte Mortalitäts- und Morbiditätsraten noch lange nach der Erkrankung. Teams der Harvard Medical School in Boston und des Wellcome Sanger Institutes in Cambridge fanden nun den Grund heraus. Die Forscher werteten dazu Blutproben von 77 Kindern aus den Niederlanden aus, die 2013 an Masern erkrankt waren. Sie nahmen jeweils eine Probe vor der und zwei Monate nach der Erkrankung und verglichen diese mit Blutproben von geimpften und nicht geimpften Kindern, die nicht an Masern erkrankt waren. Mit dem sog. VirScan-Verfahren untersuchten sie das Blut der Kinder auf spezifische Antikörper, die das Immunsystem bildet, wenn der Körper durch eine Erkrankung oder Imp- fung mit bestimmten Erregern in Kontakt kommt. Dabei konnten sie nachweisen, dass die Kinder nach einer Maserninfektion im Durchschnitt 40\% ihrer spezifischen Antikörper verloren hatten. Das erklärt die geschwächte Immunabwehr nach der Masernerkrankung und die Anfälligkeit für andere Infektionen. Die Viren regten die Bildung neuer, naiver Gedächtniszellen an, die noch keine abzuwehrenden Erreger kannten.

Wie dieses Löschen des Immungedächtnisses funktioniert, untersuchte das zweite Forscherteam mit einer Genanalyse von BZellen aus Blutproben von an Masern erkrankten Kindern. Diese Gedächtniszellen steuern bei ihnen bekannten Erregern die Produktion der passenden Antikörper. Die Analyse ergab, dass die Masernviren nach einer Infektion B-Gedächtniszellen zerstören, die bereits auf Erreger programmiert waren. So geht auch ein bestehender Impfschutz gegen andere Erkrankungen wie z.B. Grippe verloren. Wie die Studien weiter ergaben, blieben Gedächtniszellen und Antikörper erhalten, wenn die Kinder bei der gängigen MMR-Impfung erstmals mit in ihrer Virulenz abgeschwächten Viren in Kontakt kamen. In diesem Fall nahmen die Abwehrzellen sogar zu. Daher plädieren die Forscher für die Impfung, da sie auch vor anderen Infektionskrankheiten schützt.

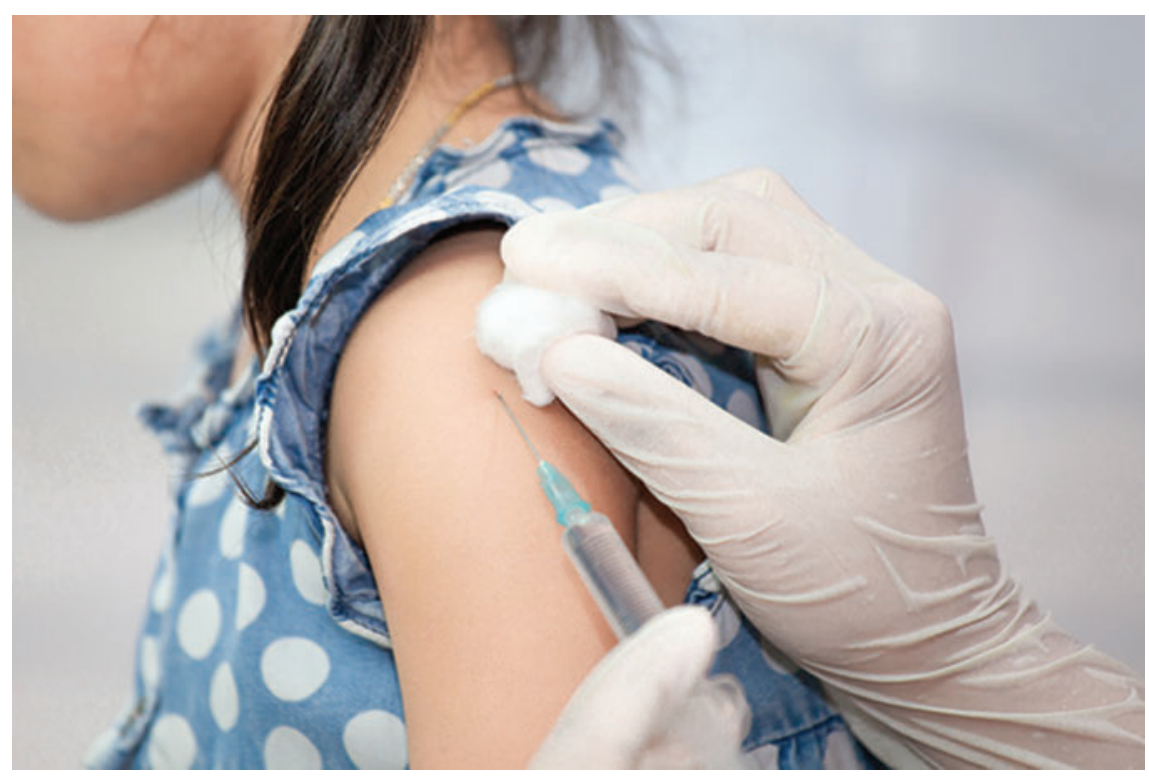

Eine Masernimpfung stärkt das Immunsystem auch gegen andere Infektionen. (Foto: pingpao-stock.adobe.com) 
Quellen: Science 2019, DOI: 10.1126/ science.aay6485 und Science Imunology 2019, DOI: 10.1126 / sciimmunol.aay6125

Prospektive Studie

\section{Striae geben Hinweis auf Verwachsungen}

Für die meisten Frauen sind Schwangerschaftsstreifen (Striae) in erster Linie ein kosmetisches Problem. Doch sie können auch wichtige medizinische Informationen geben, wie ein Team um den Gynäkologen Dr. Ozgur Kan von der türkischen Universität Hitit in Corum herausgefunden hat. In einer prospektiven Studie wurden 100 Frauen mit einer Re-Sectio und vergleichbaren demografischen Faktoren in drei Gruppen eingeteilt, jeweils abhängig von der Intensität der Striae. Mit dem sog. Davey Score wurde ein Zusammenhang zwischen Striae und inneren Verwachsungen ermittelt. Mit diesem Score wird der Bauch mit einer gedachten Mittellinie und einer horizontalen Linie durch den Bauchnabel in vier Quadranten geteilt. In jedem Quadranten wird sowohl die Menge (0-2) als auch die Schwere ( 0 = abwesend, 1-2 = leicht, 3-8=schwer) der Striae beurteilt. Es wurde besonders in den beiden oberen Quadranten ein hoch signifikanter Zusammenhang zwischen der Dichte der Striae und der Intensität der Adhäsionen festgestellt. Das Forscherteam sieht in der präoperativen Beurteilung von Striae ein gutes Instrument, um sich bei einer möglichen Re-Sectio auf Adhäsionen vorzubereiten.

Quelle: Journal of Obstetrics and Gynaecology Research 2019; 45 (12), DOI: https: //doi.org/10.1111/jog.14125

\section{Kohortenstudie}

\section{Geburtsmodus und späteres Übergewicht}

Eine schwedische Kohortenstudie widerlegt einen Zusammenhang zwischen Sectio und späterer Adipositas, den zahlreiche Studien zuvor herausgefunden

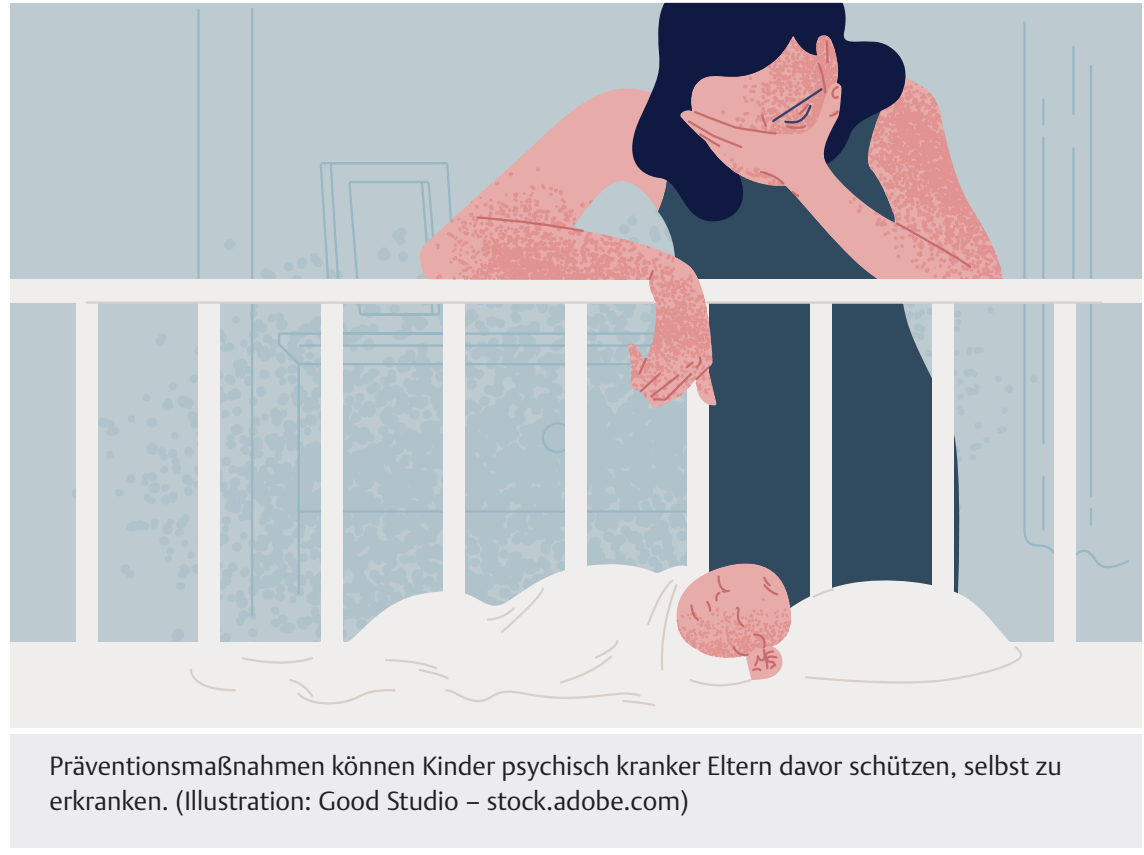

hatten. Das Forscherteam des Public Health Departments des Stockholmer Karolinska Instituts untersuchte die Daten von rund 98000 Männern aus dem schwedischen Bevölkerungsregister-von ihrer Geburt in den Jahren 1982 bis 1987 bis zum Alter von 18 Jahren. Dazu wurden sie je nach Geburtsmodus in drei Gruppen eingeteilt: vaginale Geburt, elektive oder sekundäre Sectio. Rund 4,9\% der gesamten Gruppe waren mit 18 Jahren adipös (BMI $\geq 30$ ). Dabei zeigten sich in den drei Gruppen nur geringfügige Unterschiede: vaginale Geburt 4,9\%, elektive Sectio 5,5\%, sekundäre Sectio 5,6\%. Berücksichtigt wurden neben dem mütterlichen BMI auch Faktoren wie Gestationsdiabetes, mütterlicher Hypertonus, Rauchen während der Schwangerschaft sowie das Alter und der Bildungsgrad der Mutter, das Geburtsgewicht und das Gestationsalter. Ebenso wurden Probanden mit Geschwistern verglichen, um eine Verzerrung durch familiäre Faktoren auszuschließen. Dennoch sehen die Forscher Schwächen in der Studie, da nur Männer untersucht wurden und nur von $42 \%$ der 18-Jährigen Daten erhoben werden konnten.

Quelle: PLOS Medicine 2019, DOI: https: //doi.org/10.1371/journal.pmed.1002996
Präventionsprojekt

Haben Eltern eine psychische Erkrankung, besteht für deren Kinder ein mehrfach erhöhtes Risiko, ebenfalls zu erkranken. Doch nicht selten sind sie symptomatisch unauffällig und funktionieren angepasst im belasteten Familienleben. Um diesen Kindern bereits präventiv Unterstützung anbieten zu können, finanziert der Inno vationsfonds des Gemeinsamen Bundesausschusses (GBA) das Projekt CHIMPS-NET (CHIMPS = Children of mentally ill parents) für 3 Jahre mit rund 6,8 Mio. Euro. Unter Federführung des Universitätsklinikums Hamburg-Eppendorf soll in jedem Bundesland ein Standort entstehen, an dem Kinder während der Behandlung ihrer Eltern auf psychische Auffälligkeiten untersucht werden können. Neben Präventionsmaßnahmen mit der Unterstützung von Sozialarbeitern sind Familientherapien bei Psychotherapeuten geplant. Für Familien in ländlichen Gebieten soll es Online-Angebote mit therapeutischer Begleitung geben. Alle drei Angebote werden in randomisiert kontrollierten Studien mit Kindern von psychisch erkrankten Eltern verglichen, die keine Unterstützung bekommen. Diese

\section{Unterstützung für Kinder psychisch kranker Eltern}




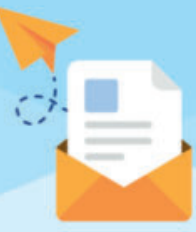

\section{CALL FOR PAPERS}

Haben Sie ein Thema, das Ihnen wichtig ist und über das Sie gern mehr lesen wollen? Möchten Sie Ihre wissenschaftliche Arbeit in der Zeitschrift DIE HEBAMME publizieren oder einen Artikel verfassen, der evidenzbasiertes Wissen und praktische Erfahrung rund um die Hebammenarbeit verbindet? Wir freuen uns über lhre Themenvorschläge und Artikeleinreichungen. Bitte richten Sie Ihre Einsendung an:

E-Mail: diehebamme@thieme.de $\mathbf{\nabla}$

Alle eingereichten Artikel gehen ins Review. Das Herausgeberteam entscheidet über die Annahme des Artikels. Sie erhalten in jedem Fall ein Feedback.

bisher fehlende Hilfe könnte nach Abschluss des Projekts in die GKV-Regelversorgung aufgenommen werden, um zu verhindern, dass psychische Erkrankungen chronifizieren.

Quelle: https: / /innovationsfonds.g-ba.de/ projekte/neue-versorgungsformen/chimpsnet-kinder-und-jugendliche-mit-psychischkranken-und-suchtkranken-eltern-childrenof-mentaly-ill-parents-network.256

Klinische Studien

\section{Abnabeln bei Sectio und Frühgeburt untersucht}

Obwohl die Weltgesundheitsorganisation (WHO) ein Abnabeln frühestens nach 60 Sekunden empfiehlt, wird bei vielen Geburten das verzögerte Abnabeln nicht praktiziert. Dazu gehören unter anderem Sectiones und Frühgeburten. Während bei Sectiones das Blutungsrisiko der Mutter im Vordergrund steht, hat bei Frühgeborenen die schnelle Versorgung des Babys Priorität. Diese beiden Szenarien wurden in zwei amerikanischen Studien untersucht.

Ein Team am Irving Medical Center der Columbia Universität in New York teilte
113 Frauen in zwei Gruppen auf: Während in der ersten Gruppe $(n=56)$ innerhalb von 15 Sekunden abgenabelt wurde, erfolgte die Abnabelung in der zweiten Gruppe $(n=57)$ erst nach 60 Sekunden. Alle 113 Frauen bekamen eine elektive Sectio am Termin. Beurteilt wurde der Hb-Wert der Mütter am ersten Tag nach der Geburt und der Hb-Wert der Neugeborenen. Bei den Müttern mit verzögerter Abnabelung war der Hb-Wert zwar niedriger als nach sofortiger Durchtrennung der Nabelschnur, dennoch war der Unterschied klinisch nicht relevant und nicht signifikant. Bei den Neugeborenen fiel der mittlere Hb-Wert nach verzögerter Abnabelung dagegen mit $18,1 \mathrm{mg} / \mathrm{dl}$ vs. $16,4 \mathrm{mg} / \mathrm{dl}$ höher aus und war mit einem 95-\%-Konfidenzintervall von 0,75 bis $2,59 \mathrm{~g} / \mathrm{dl}$ signifikant.

In einer zweiten Studie, der internationalen PREMOD2-Studie (an der in Deutschland die Universität Ulm beteiligt war), sollte herausgefunden werden, ob das Ausstreichen der Nabelschnur bei Frühgeborenen<32 SSW den Zustand der Babys verbessert. An der Studie sollten 1200 Frühgeborene teilnehmen, bei allen sollte verzögert abgenabelt werden, aber nur bei der einen Hälfte sollte die Nabelschnur zusätzlich ausgestrichen werden. Nach dem Einschluss von 474 Babys musste die Studie abgebrochen werden, da es bei $12 \%$ der Babys nach dem Ausstreichen der Nabelschnur zum Tod oder zu einer Hirnblutung gekommen war.

Quellen: JAMA 2019; 322(19): 1869-1876, DOI:10.1001/jama.2019.15995 und JAMA 2019; 322(19): 1877-1886, DOI:10.1001/ jama.2019.16004

EVIDENZ ZUM VERZÖGERTEN ABNABELN

Zusammenfassend lassen sich die oben zitierten Studien nicht miteinander vergleichen. Zum einen unterscheiden sich die jeweils untersuchten Gruppen stark in ihren Risikofaktoren. Während die erste Studie Neugeborene am Termin untersuchte, wurden in der zweiten Studie ausschließlich Frühgeborene $<32$ SSW betrachtet.
Zum anderen kann das Vorgehen des verzögerten Abnabelns nicht mit dem Ausstreichen der Nabelschnur (Cord milking) gleichgesetzt werden ( $\rightarrow$ einen Artikel dazu finden Sie in unserem Online-Archiv in DIE HEBAMME 4/2019 unter www.thieme-connect.de $\rightarrow$ Kalbér A, Kühn T. Verzögertes Abnabeln - Frauen kompetent beraten. Die Hebamme 2019; 32 (4): 23-31).

Fakt ist: Gegenwärtig gibt es keine ausreichende Evidenz, um die Methode des Ausstreichens der Nabelschnur, insbesondere bei sehr unreifen Frühgeborenen, eindeutig zu unterstützen oder zu widerlegen. Das verzögerte Abnabeln hingegen ist eine einfach zu etablierende, kostenneutrale und evidenzbasierte Maßnahme mit zahlreichen gesundheitlichen Vorteilen für alle Neugeborenen bei allen Geburten (Quelle: WHO 2014, ERC 2015).

Die nachfolgende Poster-Grafik zum

Thema „Abwarten ...“ vermittelt auf einen Blick, was den optimalen Zeitpunkt ausmacht und welche Vorteile ein spätes Abnabeln für das Neugeborene und eine frauenorientierte Betreuung bietet. Nutzen Sie diese gerne in Ihrer Klinik oder in Ihrem Geburtshaus ( $\vee$ Seite 8 ). 


\section{Abwarten...}

\section{...bevor nach den Klemmen gegriffen wird!}

$\rightarrow$ Wurde der Zustand des Kindes überprüft?

$\rightarrow$ Hat das Kind so viel Nabelschnurblut wie möglich erhalten?

$\rightarrow$ Wurde es im Team abgesprochen?

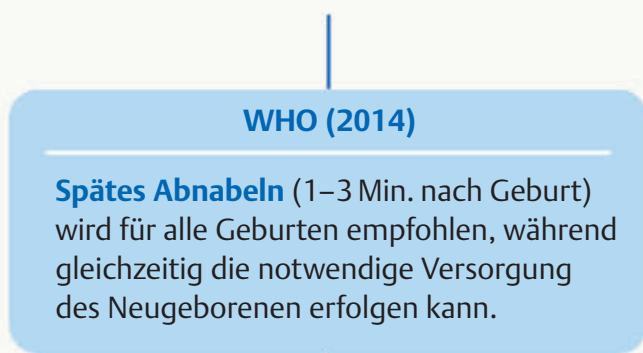

\section{ERC (2015)}

Frühes Abnabeln (<1 Min. nach Geburt) wird nicht empfohlen, außer das Neugeborene ist asphyktisch und muss für eine sofortige Reanimation ( $\mathrm{HF}<100$ spm) umgelagert werden.

\section{In Nabelschnur und} Plazenta befinden sich bis zu $30 \%$ des kindlichen Blutvolumens!

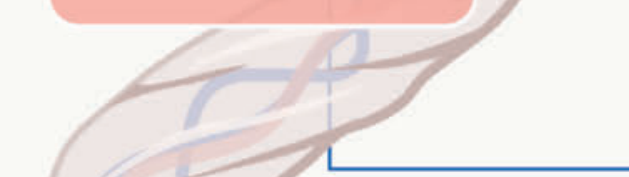

Autorinnen: Gemma Dicksinson,

Daniela Garten, Anna Hutsch (2019).

Bildgestaltung: Thieme Gruppe
... fördert die neurologische Entwicklung, reduziert Zerebralparesen und Lernstörungen.

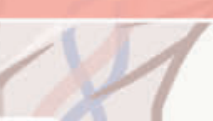

... reduziert das Risiko von Anämie, Bluttransfusion, nekrotisierender Enterokolitis und Hirnblutungen. $\rightarrow$ Etwa $90 \%$ aller Kinder werden in gutem Zustand geboren und benötigen keinerlei Reanimationsmaßnahmen.

$\rightarrow$ Optimales Abnabeln ist vor allem für Frühgeborene von Vorteil.

$\rightarrow$ Uterotonische Medikamente können bereits während des Auspulsierens verabreicht werden.

$\rightarrow$ Die Abnahme der Blutgasanalyse ist auch bei intakter Nabelschnur möglich.

$\rightarrow$ Optimales Abnabeln kann das Geburtserlebnis der Frau bedeutend verbessern.

\section{Optimales/Spätes Abnabeln}

Ermöglicht neben allen oben genannten Vorteilen, den besten Start ins Leben eines Neugeborenen, eine frauenorientierte Betreuung und trägt zur mentalen und emotionalen Gesundheit von Familien bei. 